\title{
Recent developments about multivalued weakly Picard operators
}

\author{
Gülhan Minak Ishak Altun Salvador Romaguera
}

\begin{abstract}
In the present paper, considering a recent technique, which is used by Wardowski [24] for fixed points of single-valued mappings, we give a new and general class of multivalued weakly Picard operators on complete metric spaces and show that the class of multivalued almost contractions (both linear and nonlinear cases in the sense of Berinde and Berinde [9]) is a proper subset of this new class. We also give a nontrivial example showing this fact.
\end{abstract}

\section{Introduction and preliminaries}

Let $(X, d)$ be a metric space, $P(X)$ be the collection of all nonempty subsets of $X$, $C B(X)$ be the collection of all nonempty closed and bounded subsets of $X$ and $K(X)$ be the collection of all nonempty compact subsets of $X$. It is well known that $H: C B(X) \times C B(X) \rightarrow \mathbb{R}$ defined by

$$
H(A, B)=\max \left\{\sup _{x \in A} D(x, B), \sup _{y \in B} D(y, A)\right\}
$$

is a metric on $C B(X)$, which is called the Pompeiu-Hausdorff metric induced by $d$, where $D(x, B)=\inf \{d(x, y): y \in B\}$. We can find detailed information about the Pompeiu-Hausdorff metric in [2,10,13]. An element $x \in X$ is said to be a fixed point of a multivalued mapping $T: X \rightarrow P(X)$ if $x \in T x$. In 1991,

Received by the editors in October 2014.

Communicated by F. Bastin.

2010 Mathematics Subject Classification : Primary 54H25; Secondary, 47H10.

Key words and phrases: Multivalued weakly Picard operator, multivalued almost F-contraction, fixed point. 
Rus [19] introduced the concept of multivalued weakly Picard (MWP) operator on a metric space $(X, d)$ as follows. A multivalued mapping $T: X \rightarrow P(X)$ is MWP operator if there exists a sequence $\left\{x_{n}\right\}$ in $X$ such that $x_{n+1} \in T x_{n}$ for any initial point $x_{0}$, which converges to a fixed point of $T$ (see $\left.[20,21]\right)$. We shall denote the class of all MWP operators on $X$ by $\mathcal{M}_{W P}(X)$.

Let $T: X \rightarrow C B(X)$. $T$ is said to be a multivalued nonlinear almost contraction [9] if there exist a constant $L \geq 0$ and a function $\varphi:[0, \infty) \rightarrow[0,1)$ satisfying

$$
\limsup _{t \rightarrow s^{+}} \varphi(t)<1, \forall s \geq 0
$$

such that

$$
H(T x, T y) \leq \varphi(d(x, y)) d(x, y)+L D(y, T x)
$$

for all $x, y \in X$. A function $\varphi:[0, \infty) \rightarrow[0,1)$ satisfying (1.1) is called MizoguchiTakahashi function $(\mathcal{M T}$-function $[11,12])$ in the literature.

Note that, by the symmetry property of the distance, the nonlinear almost contractive condition implicitly includes the following dual one

$$
H(T x, T y) \leq \varphi(d(x, y)) d(x, y)+L D(x, T y)
$$

for all $x, y \in X$. So, in order to check the nonlinear almost contractiveness of a multivalued mapping $T$, it is necessary to check both (1.2) and (1.3). Specially, if $\varphi:[0, \infty) \rightarrow[0,1)$ is a constant, then $T$ is called multivalued almost contraction. If $L=0$, then (1.2) turns to the famous Mizoguchi-Takahashi [14] contractive condition, which includes the multivalued contractions in sense of Nadler [16]. We denote show the class of all multivalued nonlinear almost contractions on $X$ by $\mathcal{M}_{N A}(X)$.

Berinde and Berinde [9] proved that if $(X, d)$ is a complete metric space and $T: X \rightarrow C B(X)$ is a multivalued nonlinear almost contraction, then $T$ has a fixed point. Analyzing the proof of this result, we can observe that $T$ is MWP operator. Therefore, if $(X, d)$ is complete then $\mathcal{M}_{N A}(X) \subset \mathcal{M}_{W P}(X)$. We can find some important information about single-valued and multivalued weakly Picard operators on complete metric space in $[5,6,7,8]$.

In this paper, by considering the recent technique of Wardowski [24], we give a new class of MWP operators, which is more general than $\mathcal{M}_{N A}(X)$ on complete metric spaces. First, we recall the Wardowski's technique.

Let $F:(0, \infty) \rightarrow \mathbb{R}$ be a function. Consider the following conditions for $F$ :

(F1) $F$ is strictly increasing, i.e., for all $\alpha, \beta \in(0, \infty)$ such that $\alpha<\beta$, $F(\alpha)<F(\beta)$,

(F2) For each sequence $\left\{\alpha_{n}\right\}$ of positive numbers

$$
\lim _{n \rightarrow \infty} \alpha_{n}=0 \text { if and only if } \lim _{n \rightarrow \infty} F\left(\alpha_{n}\right)=-\infty,
$$

(F3) There exists $k \in(0,1)$ such that $\lim _{\alpha \rightarrow 0^{+}} \alpha^{k} F(\alpha)=0$,

(F4) $F(\inf A)=\inf F(A)$ for all $A \subset(0, \infty)$ with inf $A>0$.

By considering the conditions (F1)-(F3), Wardowski [24] introduced the concept of $F$-contractions, which more general than ordinary contraction, for single valued maps and show that every $F$-contraction on a complete metric space has a unique fixed point. (See [24] for more detailed information about $F$-contractions). Then, considering this new concept, some studies have been made. 
Remark 1. In [23] Udo-Utun shows that F-contractions are closely related to $(\delta, k)$-weak contractions (almost contractions) in the sense of Berinde. Therefore in Theorem 2.1 (ii) of [23], it has been shown that every F-contraction $T$ is a $(\delta, k)$-weak contraction, where $T$ is a self map of a bounded closed and convex subset of a real Banach space. But we know that this two concepts are independent on a (complete) metric space as shown in the following two examples. We can find them in [24] and in [18], respectively.

In the following example, the mapping $T$ is not $(\delta, k)$-weak contraction, but it is F-contraction on a complete metric space.

Example 1. Let $X=\left\{x_{n}=\frac{n(n+1)}{2}: n \in \mathbb{N}\right\}$ and $d(x, y)=|x-y|$. Then $(X, d)$ is $a$ complete metric space. Define a mapping $T: X \rightarrow X$ by:

$$
T x=\left\{\begin{array}{ll}
x_{1}, & x=x_{1} \\
x_{n-1}, & x=x_{n}
\end{array} .\right.
$$

Then, as shown in Example 2.5 of [24], $T$ is F-contraction with $F(\alpha)=\alpha+\ln \alpha$ and $\tau=1$.

On the other hand, since $d\left(x_{n-1}, T x_{n}\right)=0$ and

$$
\lim _{n \rightarrow \infty} \frac{d\left(T x_{n}, T x_{n-1}\right)}{d\left(x_{n}, x_{n-1}\right)}=\lim _{n \rightarrow \infty} \frac{2 n-2}{2 n}=1,
$$

then we can not find $\delta \in(0,1)$ and $k \geq 0$ satisfying

$$
d(T x, T y) \leq \delta d(x, y)+k d(y, T x)
$$

for all $x, y \in X$. Therefore, $T$ is not $a(\delta, k)$-weak contraction.

In the following example, the mapping $T$ is $(\delta, k)$-weak contraction, but it is not $F$-contraction on a complete metric space.

Example 2. Let $X=[0,2]$ with the usual metric and $T: X \rightarrow X$ be defined by

$$
T x=\left\{\begin{array}{ll}
\frac{x}{2}, & x \in[0,1) \\
2, & x \in[1,2]
\end{array} .\right.
$$

Then $T$ is $a(\delta, k)$-weak contraction with $\delta=\frac{1}{2}$ and $k=3$ (Example 1.3 .19 of [18]). But, since $T$ is not continuous and so it is not an F-contraction.

We denote by $\mathcal{F}$ and $\mathcal{F}_{*}$ the set of all functions $F$ satisfying (F1)-(F3) and (F1)-(F4), respectively. It is clear that $\mathcal{F}_{*} \subset \mathcal{F}$. Some examples of functions belonging $\mathcal{F}_{*}$ are $F_{1}(\alpha)=\ln \alpha, F_{2}(\alpha)=\alpha+\ln \alpha, F_{3}(\alpha)=-\frac{1}{\sqrt{\alpha}}$ and $F_{4}(\alpha)=$ $\ln \left(\alpha^{2}+\alpha\right)$. If we define $F_{5}(\alpha)=\ln \alpha$ for $\alpha \leq 1$ and $F_{5}(\alpha)=2 \alpha$ for $\alpha>1$, then $F_{5} \in \mathcal{F} \backslash \mathcal{F}_{*}$. If $F$ is nondecreasing, then it satisfies (F4) if and only if it is right continuous.

Later, the technique of Wardowski was used for multivalued mappings by many authors (see [1, 3, 4, 15, 17, 22]). Here, we shall mentioned some important of them. 
Theorem 1 ([17]). Let $(X, d)$ be a complete metric space and $T: X \rightarrow C B(X)$. If there exist $F \in \mathcal{F}_{*}$ and $\tau:(0, \infty) \rightarrow(0, \infty)$ such that

$$
\liminf _{t \rightarrow s^{+}} \tau(t)>0, \forall s \geq 0,
$$

satisfying

$$
\tau(d(x, y))+F(H(T x, T y)) \leq F(d(x, y))
$$

for all $x, y \in X$ with $H(T x, T y)>0$. Then $T$ has a fixed point in $X$.

Theorem 2 ([3]). Let $(X, d)$ be a complete metric space and $T: X \rightarrow C B(X)$. If $T$ is a multivalued almost $F$-contraction with $F \in \mathcal{F}_{*}$, that is, there exist two constants $\tau>0$ and $\lambda \geq 0$ satisfying

$$
\tau+F(H(T x, T y)) \leq F((d(x, y)+\lambda D(y, T x))
$$

for all $x, y \in X$ with $H(T x, T y)>0$, then $T$ has a fixed point in $X$.

If we examine the proofs of Theorem 1 and Theorem 2, we can see that the mentioned maps belong to $\mathcal{M}_{W P}(X)$. In Theorem 1 (or in Theorem 2), the condition (F4) on $F$ cannot be removed as shown in Example 1 of [3]. However, if we take $T: X \rightarrow K(X)$ in these theorems, we can remove the condition (F4) on $F$.

\section{Main result}

Our main results are based on the following new definition.

Definition 1. Let $(X, d)$ be a metric space and $T: X \rightarrow C B(X)$. We say that $T$ is a multivalued nonlinear almost $F$-contraction with $F \in \mathcal{F}$ if there exist a constant $\lambda \geq 0$ and a function $\tau:(0, \infty) \rightarrow(0, \infty)$ such that

$$
\liminf _{t \rightarrow s^{+}} \tau(t)>0, \text { for all } s \geq 0
$$

satisfying

$$
\tau(d(x, y))+F(H(T x, T y)) \leq F((d(x, y)+\lambda D(y, T x))
$$

for all $x, y \in X$ with $H(T x, T y)>0$.

We shall denote the class of all multivalued nonlinear almost $F$-contractions on $X$ by $\mathcal{M}_{N A F}(X)$.

Remark 2. Taking $\tau(t)=\tau>0$ in Definition 1, we deduce that every multivalued almost F-contraction is also multivalued nonlinear almost F-contraction.

Remark 3. Every multivalued nonlinear almost contraction is also multivalued nonlinear almost $F$-contraction with a special $F$, that is, $\mathcal{M}_{N A}(X) \subset \mathcal{M}_{N A F}(X)$. Indeed, let $(X, d)$ be metric space and $T \in \mathcal{M}_{N A}(X)$. Then, there exist a constant $L \geq 0$ and an $\mathcal{M T}$-function $\varphi$ satisfying

$$
H(T x, T y) \leq \varphi(d(x, y)) d(x, y)+L D(y, T x)
$$


for all $x, y \in X$. Define $\beta(t)=\frac{1+\varphi(t)}{2}$, then $\beta$ is also an $\mathcal{M T}$-function. Therefore from (2.2),

$$
\begin{aligned}
H(T x, T y) & \leq \varphi(d(x, y)) d(x, y)+L D(y, T x) \\
& \leq \frac{1+\varphi(d(x, y))}{2} d(x, y)+L[1+\varphi(d(x, y))] D(y, T x) \\
& =\beta(d(x, y)) d(x, y)+2 L \beta(d(x, y)) D(y, T x) \\
& =\beta(d(x, y))[d(x, y)+2 L D(y, T x)]
\end{aligned}
$$

for all $x, y \in X$ with $H(T x, T y)>0$. Thus, we get

$$
-\ln (\beta(d(x, y)))+\ln (H(T x, T y)) \leq \ln (d(x, y)+2 L D(y, T x))
$$

for all $x, y \in X$ with $H(T x, T y)>0$. Now, define $\tau(t)=-\ln \beta(t)$. Since $\beta$ is an $\mathcal{M T}$-function, then

$$
\liminf _{t \rightarrow s^{+}} \tau(t)>0 \text { for all } s \geq 0 .
$$

Therefore, from (2.3), $T$ is a multivalued nonlinear almost F-contraction with $F(\alpha)=\ln \alpha, \lambda=2 L$ and $\tau(t)=-\ln \left(\frac{1+\varphi(t)}{2}\right)$, that is $T \in \mathcal{M}_{N A F}(X)$.

Theorem 3. Let $(X, d)$ be a complete metric space and $T \in \mathcal{M}_{N A F}(X)$ with $F \in \mathcal{F}_{*}$, then $T \in \mathcal{M}_{W P}(X)$.

Proof. Let $x_{0} \in X$. As $T x$ is nonempty for all $x \in X$, we can choose $x_{1} \in T x_{0}$. If $x_{1} \in T x_{1}$, then $x_{1}$ is a fixed point of $T$. In this case, we construct a sequence $\left\{x_{n}\right\}$ by $x_{n}=x_{1}$ for $n \geq 1$, then $x_{n+1} \in T x_{n}$ and $\left\{x_{n}\right\}$ converges to a fixed point of $T$, that is $T \in \mathcal{M}_{W P}(X)$. Now, suppose $x_{1} \notin T x_{1}$. Then, as $T x_{1}$ is closed, $D\left(x_{1}, T x_{1}\right)>0$. On the other hand, as $D\left(x_{1}, T x_{1}\right) \leq H\left(T x_{0}, T x_{1}\right)$, from (F1) we have

$$
F\left(D\left(x_{1}, T x_{1}\right)\right) \leq F\left(H\left(T x_{0}, T x_{1}\right)\right) .
$$

Since $T \in \mathcal{M}_{N A F}(X)$, we have

$$
\begin{aligned}
F\left(D\left(x_{1}, T x_{1}\right)\right) & \leq F\left(H\left(T x_{0}, T x_{1}\right)\right) \\
& \leq F\left(d\left(x_{1}, x_{0}\right)+\lambda D\left(x_{1}, T x_{0}\right)\right)-\tau\left(d\left(x_{1}, x_{0}\right)\right) \\
& =F\left(d\left(x_{1}, x_{0}\right)\right)-\tau\left(d\left(x_{1}, x_{0}\right)\right) .
\end{aligned}
$$

From (F4) we can write (note that $D\left(x_{1}, T x_{1}\right)>0$ )

$$
F\left(D\left(x_{1}, T x_{1}\right)\right)=\inf _{y \in T x_{1}} F\left(d\left(x_{1}, y\right)\right),
$$

and so from (2.4) we have

$$
\begin{aligned}
\inf _{y \in T x_{1}} F\left(d\left(x_{1}, y\right)\right) & \leq F\left(d\left(x_{1}, x_{0}\right)\right)-\tau\left(d\left(x_{1}, x_{0}\right)\right) \\
& <F\left(d\left(x_{1}, x_{0}\right)\right)-\frac{\tau\left(d\left(x_{1}, x_{0}\right)\right)}{2} .
\end{aligned}
$$

Then, from (2.5) there exists $x_{2} \in T x_{1}$ such that

$$
F\left(d\left(x_{1}, x_{2}\right)\right) \leq F\left(d\left(x_{1}, x_{0}\right)\right)-\frac{\tau\left(d\left(x_{1}, x_{0}\right)\right)}{2} .
$$


If $x_{2} \in T x_{2}$ we are finished. Otherwise, by the same way we can find $x_{3} \in T x_{2}$ such that

$$
F\left(d\left(x_{2}, x_{3}\right)\right) \leq F\left(d\left(x_{2}, x_{1}\right)\right)-\frac{\tau\left(d\left(x_{2}, x_{1}\right)\right)}{2} .
$$

By induction, we can find a sequence $\left\{x_{n}\right\}$ in $X$ such that $x_{n+1} \in T x_{n}$ (we may assume $\left.x_{n} \notin T x_{n}\right)$ and

$$
F\left(d\left(x_{n}, x_{n+1}\right)\right) \leq F\left(d\left(x_{n}, x_{n-1}\right)\right)-\frac{\tau\left(d\left(x_{n}, x_{n-1}\right)\right)}{2}
$$

for all $n \in \mathbb{N}$. Denote $a_{n}=d\left(x_{n}, x_{n+1}\right)$ for $n \in \mathbb{N}$, then $a_{n}>0$ and from (2.6) $\left\{a_{n}\right\}$ is decreasing. Therefore there exists $\delta \geq 0$ such that $\lim _{n \rightarrow \infty} a_{n}=\delta$. Now let $\delta>0$. Using (2.6), the following holds:

$$
\begin{aligned}
F\left(a_{n}\right) \leq & F\left(a_{n-1}\right)-\frac{\tau\left(a_{n-1}\right)}{2} \\
\leq & F\left(a_{n-2}\right)-\frac{\tau\left(a_{n-1}\right)}{2}-\frac{\tau\left(a_{n-2}\right)}{2} \\
& \vdots \\
\leq & F\left(a_{0}\right)-\frac{\tau\left(a_{n-1}\right)+\tau\left(a_{n-2}\right)+\cdots+\tau\left(a_{0}\right)}{2} .
\end{aligned}
$$

Let $p_{n}$ be a greatest number in $\{0,1, \cdots, n-1\}$ such that

$$
\tau\left(a_{p_{n}}\right)=\min \left\{\tau\left(a_{0}\right), \tau\left(a_{1}\right), \cdots, \tau\left(a_{n-1}\right)\right\}
$$

for all $n \in \mathbb{N}$. In this case, $\left\{p_{n}\right\}$ is a nondecreasing sequence. From (2.7), we get

$$
F\left(a_{n}\right) \leq F\left(a_{0}\right)-\frac{n}{2} \tau\left(a_{p_{n}}\right) .
$$

Now consider the sequence $\left\{\tau\left(a_{p_{n}}\right)\right\}$. We distinguish two cases.

Case 1. For each $n \in \mathbb{N}$ there is $m>n$ such that $\tau\left(a_{p_{n}}\right)>\tau\left(a_{p_{m}}\right)$. Then we obtain a subsequence $\left\{a_{p_{n_{k}}}\right\}$ of $\left\{a_{p_{n}}\right\}$ with $\tau\left(a_{p_{n_{k}}}\right)>\tau\left(a_{p_{n_{k+1}}}\right)$ for all $k$. Since $a_{p_{n_{k}}} \rightarrow \delta^{+}$we deduce that

$$
\liminf _{k \rightarrow \infty} \tau\left(a_{p_{n_{k}}}\right)>0 .
$$

Hence $F\left(a_{n_{k}}\right) \leq F\left(a_{0}\right)-\frac{n_{k}}{2} \tau\left(a_{p_{n_{k}}}\right)$ for all $k$. Consequently $\lim _{k \rightarrow \infty} F\left(a_{n_{k}}\right)=-\infty$, and by (F2), $\lim _{k \rightarrow \infty} a_{p_{n_{k}}}=0$, which contradicts that $\lim _{n \rightarrow \infty} a_{n}>0$.

Case 2. There is $n_{0} \in \mathbb{N}$ such that $\tau\left(a_{p_{n_{0}}}\right)=\tau\left(a_{p_{m}}\right)$ for all $m>n_{0}$. Then $F\left(a_{m}\right) \leq F\left(a_{0}\right)-\frac{m}{2} \tau\left(a_{p_{n_{0}}}\right)$ for all $m>n_{0}$. Hence $\lim _{m \rightarrow \infty} F\left(a_{m}\right)=-\infty$, so $\lim _{m \rightarrow \infty} a_{m}=0$, which contradicts that $\lim _{m \rightarrow \infty} a_{m}>0$.

Thus $\lim _{n \rightarrow \infty} a_{n}=0$. From (F3), there exists $k \in(0,1)$ such that

$$
\lim _{n \rightarrow \infty} a_{n}^{k} F\left(a_{n}\right)=0
$$

By (2.7), the following holds for all $n \in \mathbb{N}$

$$
a_{n}^{k} F\left(a_{n}\right)-a_{n}^{k} F\left(a_{0}\right) \leq-a_{n}^{k} \frac{n}{2} \tau\left(a_{p_{n}}\right) \leq 0,
$$


that is,

$$
0 \leq a_{n}^{k} \frac{n}{2} \tau\left(a_{p_{n}}\right) \leq a_{n}^{k} F\left(a_{0}\right)-a_{n}^{k} F\left(a_{n}\right) .
$$

Letting $n \rightarrow \infty$ in (2.9), we obtain that

$$
\lim _{n \rightarrow \infty} a_{n}^{k} \frac{n}{2} \tau\left(a_{p_{n}}\right)=0
$$

Since

$$
\alpha:=\liminf _{n \rightarrow \infty} \tau\left(a_{p_{n}}\right)>0,
$$

then there exists $n_{0} \in \mathbb{N}$ such that $\tau\left(a_{p_{n}}\right)>\frac{\alpha}{2}$ for all $n \geq n_{0}$. Thus

$$
a_{n}^{k} \frac{n \alpha}{4} \leq a_{n}^{k} \frac{n}{2} \tau\left(a_{p_{n}}\right)
$$

for all $n \geq n_{0}$. Letting $n \rightarrow \infty$ in (2.11), we obtain that

$$
0 \leq \lim _{n \rightarrow \infty} a_{n}^{k} \frac{n \alpha}{4} \leq \lim _{n \rightarrow \infty} a_{n}^{k} \frac{n}{2} \tau\left(a_{p_{n}}\right)=0,
$$

that is

$$
\lim _{n \rightarrow \infty} n a_{n}^{k}=0 .
$$

From (2.12), there exits $n_{1} \in \mathbb{N}$ such that $n a_{n}^{k} \leq 1$ for all $n \geq n_{1}$. So, we have, for all $n \geq n_{1}$

$$
a_{n} \leq \frac{1}{n^{\frac{1}{k}}} .
$$

In order to show that $\left\{x_{n}\right\}$ is a Cauchy sequence consider $m, n \in \mathbb{N}$ such that $m>n \geq n_{1}$. Using the triangular inequality for the metric and from (2.13), we have

$$
\begin{aligned}
d\left(x_{n}, x_{m}\right) & \leq d\left(x_{n}, x_{n+1}\right)+d\left(x_{n+1}, x_{n+2}\right)+\cdots+d\left(x_{m-1}, x_{m}\right) \\
& =a_{n}+a_{n+1}+\cdots+a_{m-1} \\
& =\sum_{i=n}^{m-1} a_{i} \leq \sum_{i=n}^{\infty} a_{i} \leq \sum_{i=n}^{\infty} \frac{1}{i^{1 / k}}
\end{aligned}
$$

By the convergence of the series $\sum_{i=1}^{\infty} \frac{1}{i^{1 / k}}$, passing to limit $n \rightarrow \infty$, we get $d\left(x_{n}, x_{m}\right) \rightarrow 0$. This yields that $\left\{x_{n}\right\}$ is a Cauchy sequence in $(X, d)$. Since $(X, d)$ is a complete metric space, there exists $z \in X$ such that $\lim _{n \rightarrow \infty} x_{n}=z$. From (2.1), for all $x, y \in X$ with $H(T x, T y)>0$, we get

$$
H(T x, T y)<d(x, y)+\lambda D(y, T x)
$$

and so

$$
H(T x, T y) \leq d(x, y)+\lambda D(y, T x)
$$

for all $x, y \in X$. Then

$$
\begin{aligned}
D\left(x_{n+1}, T z\right) & \leq H\left(T x_{n}, T z\right) \\
& \leq d\left(x_{n}, z\right)+\lambda D\left(z, T x_{n}\right) \\
& \leq d\left(x_{n}, z\right)+\lambda d\left(z, x_{n+1}\right)
\end{aligned}
$$

Passing to limit $n \rightarrow \infty$, we obtain $D(z, T z)=0$. Thus, we get $z \in \overline{T z}=T z$. Therefore $T \in \mathcal{M}_{W P}(X)$. 
Remark 4. If we take $T: X \rightarrow K(X)$ in Theorem 3, we can remove the condition (F4) on F. Indeed, let $x_{0} \in X$ and $x_{1} \in T x_{0}$. If $x_{1} \in T x_{1}$, then the proof is complete. Let $x_{1} \notin T x_{1}$. Then, as $T x_{1}$ is closed, $D\left(x_{1}, T x_{1}\right)>0$. On the other hand, as $D\left(x_{1}, T x_{1}\right) \leq$ $H\left(T x_{0}, T x_{1}\right)$, from (F1) we have

$$
F\left(D\left(x_{1}, T x_{1}\right)\right) \leq F\left(H\left(T x_{0}, T x_{1}\right)\right)
$$

From (2.1), we can write that

$$
\begin{aligned}
F\left(D\left(x_{1}, T x_{1}\right)\right) & \leq F\left(H\left(T x_{0}, T x_{1}\right)\right) \\
& \leq F\left(d\left(x_{1}, x_{0}\right)+\lambda D\left(x_{1}, T x_{0}\right)\right)-\tau\left(d\left(x_{1}, x_{0}\right)\right) \\
& =F\left(d\left(x_{1}, x_{0}\right)\right)-\tau\left(d\left(x_{1}, x_{0}\right)\right) .
\end{aligned}
$$

Since $T x_{1}$ is compact, there exists $x_{2} \in T x_{1}$ such that $d\left(x_{1}, x_{2}\right)=D\left(x_{1}, T x_{1}\right)$. Then from (2.14) we have

$$
F\left(d\left(x_{1}, x_{2}\right)\right) \leq F\left(d\left(x_{1}, x_{0}\right)\right)-\tau\left(d\left(x_{1}, x_{0}\right)\right) .
$$

The rest of the proof can be completed as in the proof of Theorem 3.

Example 3. Consider the complete metric space $(X, d)$, where $X=\left\{\frac{1}{n^{2}}: n \in \mathbb{N}\right.$, $n \geq 2\} \cup\{0\}$ and $d: X \times X \rightarrow[0, \infty)$ is given by $d(x, y)=|x-y|$. Define $T: X \rightarrow$ $C B(X)$ and $F:(0, \infty) \rightarrow \mathbb{R}$ by

$$
T x=\left\{\begin{array}{cc}
\left\{0, \frac{1}{(n+1)^{2}}\right\}, & x=\frac{1}{n^{2}}, n>2 \\
\{x\} & , x \in\left\{0, \frac{1}{4}\right\}
\end{array}\right.
$$

and

$$
F(\alpha)=\left\{\begin{array}{cc}
\frac{\ln \alpha}{\sqrt{\alpha}}, & 0<\alpha<e^{2} \\
\frac{2 \alpha}{e^{3}}, & \alpha \geq e^{2}
\end{array},\right.
$$

respectively. We can see that $F \in \mathcal{F}_{*}$ and $\sup _{x, y \in X} d(x, y)=\frac{1}{4}<e^{2}$.

Since $H\left(T 0, T \frac{1}{4}\right)=\frac{1}{4}=d\left(0, \frac{1}{4}\right)$, then for all $F \in \mathcal{F}_{*}$ and $\tau:(0, \infty) \rightarrow(0, \infty)$ satisfying inequality (1.4), we have

$$
\tau\left(d\left(0, \frac{1}{4}\right)\right)+F\left(H\left(T 0, T \frac{1}{4}\right)\right)>F\left(d\left(0, \frac{1}{4}\right)\right) .
$$

Therefore Theorem 1 can not be applied to this example.

Now we show that $T$ is not a multivalued nonlinear almost contraction. Indeed, suppose that there exist a constant $L \geq 0$ and a $\mathcal{M T}$-function $\varphi$ satisfying (1.2). Therefore, for $x=\frac{1}{n^{2}}$ and $y=\frac{1}{(n+1)^{2}}$, then $\bar{D}(y, T x)=0$,

$$
H(T x, T y)=\frac{2 n+3}{(n+1)^{2}(n+2)^{2}} \text { and } d(x, y)=\frac{2 n+1}{n^{2}(n+1)^{2}} \text {. }
$$


Thus

$$
\begin{aligned}
& H(T x, T y) \leq \varphi(d(x, y)) d(x, y)+L D(y, T x) \\
\Leftrightarrow & \frac{2 n+3}{(n+1)^{2}(n+2)^{2}} \leq \varphi\left(\frac{2 n+1}{n^{2}(n+1)^{2}}\right) \frac{2 n+1}{n^{2}(n+1)^{2}} \\
\Leftrightarrow & \frac{(2 n+3) n^{2}}{(2 n+1)(n+2)^{2}} \leq \varphi\left(\frac{2 n+1}{n^{2}(n+1)^{2}}\right) .
\end{aligned}
$$

Taking limit supremum as $n \rightarrow \infty$, we have

$$
1 \leq \limsup _{n \rightarrow \infty} \varphi\left(\frac{2 n+1}{n^{2}(n+1)^{2}}\right) \leq \limsup _{t \rightarrow 0^{+}} \varphi(t)<1,
$$

which is a contradiction. Therefore $T$ is not multivalued nonlinear almost contraction and so $T \notin \mathcal{M}_{N A}(X)$.

On the other hand, $T \in \mathcal{M}_{N A F}(X)$ with $\lambda=1$ and $\tau=\ln \frac{100}{81}$. To see this have to show that

$$
\ln \frac{100}{81}+F(H(T x, T y)) \leq F(d(x, y)+\min \{D(y, T x), D(x, T y)\}),
$$

for all $x, y \in X$ with $H(T x, T y)>0$. First, observe that if $H(T x, T y)>0$, then $x \neq y$.

Case 1: For $x=\frac{1}{n^{2}}$ and $y=\frac{1}{m^{2}}$ with $m>n>2$, we obtain

$$
\begin{aligned}
& H(T x, T y)^{\frac{1}{\sqrt{H(T x, T y)}}} d(x, y)^{-\frac{1}{\sqrt{d(x, y)}}} \\
& \left.=\left(\frac{1}{(n+1)^{2}}-\frac{1}{(m+1)^{2}}\right)\right)^{\frac{1}{\sqrt{\frac{1}{(n+1)^{2}}-\frac{1}{(m+1)^{2}}}}}\left(\frac{1}{n^{2}}-\frac{1}{m^{2}}\right)^{-\frac{1}{\sqrt{\frac{1}{n^{2}-\frac{1}{m^{2}}}}}} \\
& =\left(\frac{(m+1)^{2}-(n+1)^{2}}{(n+1)^{2}(m+1)^{2}}\right)^{\frac{(n+1)(m+1)}{\sqrt{(m+1)^{2}-(n+1)^{2}}}}\left(\frac{m^{2}-n^{2}}{n^{2} m^{2}}\right)^{-\frac{n m}{\sqrt{m^{2}-n^{2}}}} \\
& =\left(\frac{(m+1)^{2}-(n+1)^{2}}{(n+1)^{2}(m+1)^{2}}\right)^{\frac{(n+1)(m+1)}{\sqrt{(m+1)^{2}-(n+1)^{2}}}-\frac{n m}{\sqrt{m^{2}-n^{2}}}}\left(\frac{(m+n+2) n^{2} m^{2}}{(m+n)(n+1)^{2}(m+1)^{2}}\right)^{\frac{n m}{\sqrt{m^{2}-n^{2}}}} .
\end{aligned}
$$

On the other hand, since

$$
\begin{gathered}
\frac{(m+1)^{2}-(n+1)^{2}}{(n+1)^{2}(m+1)^{2}} \leq \frac{1}{2} \\
\frac{(n+1)(m+1)}{\sqrt{(m+1)^{2}-(n+1)^{2}}}-\frac{n m}{\sqrt{m^{2}-n^{2}}} \geq 1 \\
\frac{(m+n+2) n^{2} m^{2}}{(m+n)(n+1)^{2}(m+1)^{2}}<1
\end{gathered}
$$

and

$$
\frac{n m}{\sqrt{m^{2}-n^{2}}}>1 \text {, }
$$

then we have

$$
H(T x, T y)^{\frac{1}{\sqrt{H(T x, T y)}}} d(x, y)^{-\frac{1}{\sqrt{d(x, y)}}} \leq \frac{1}{2}<\frac{81}{100} .
$$


Thus we obtain

$$
\begin{aligned}
\ln \frac{100}{81}+F(H(T x, T y)) & \leq F(d(x, y)) \\
& \leq F(d(x, y)+\min \{D(y, T x), D(x, T y)\}),
\end{aligned}
$$

that is (2.15) is satisfied.

Case 2. For $x=\frac{1}{n^{2}}, n>2$ and $y=0$, we obtain

$$
\begin{aligned}
H(T x, T y)^{\frac{1}{\sqrt{H(T x, T y)}}} d(x, y)^{-\frac{1}{\sqrt{d(x, y)}}} & =\left(\frac{1}{(n+1)^{2}}\right)^{\frac{1}{\sqrt{\frac{1}{(n+1)^{2}}}}}\left(\frac{1}{n^{2}}\right)^{-\frac{1}{\sqrt{\frac{1}{n^{2}}}}} \\
& =\frac{n^{2 n}}{(n+1)^{2(n+1)}} \\
& =\left(\frac{n}{n+1}\right)^{2 n}\left(\frac{1}{n+1}\right)^{2} \\
& <\frac{1}{2}
\end{aligned}
$$

and so (2.15) is satisfied.

Case 3. For $x=\frac{1}{4}$ and $y=0$, since $H(T x, T y)=d(x, y)=\min \{D(y, T x)$, $D(x, T y)\}=\frac{1}{4}$, we obtain

$$
\begin{aligned}
& H(T x, T y)^{\frac{1}{\sqrt{H(T x, T y)}}}[d(x, y)+\min \{d(y, T x), d(x, T y)\}]^{-\frac{1}{\sqrt{d(x, y)+\min \{d(y, T x), d(x, T y)\}}}} \\
= & \left(\frac{1}{4}\right)^{2}\left(\frac{1}{2}\right)^{-\sqrt{2}}<\frac{1}{16} \cdot 4=\frac{1}{4}<\frac{1}{2}
\end{aligned}
$$

that is (2.15) is satisfied.

Case 4 . For $x=\frac{1}{n^{2}}, n>2$ and $y=\frac{1}{4}$, since $H(T x, T y)=\frac{1}{4}$ and

$$
d(x, y)=\min \{D(y, T x), D(x, T y)\}=\frac{1}{4}-\frac{1}{n^{2}},
$$

since

for $n \geq 3$, we obtain

$$
\frac{1}{2}-\frac{2}{n^{2}} \geq \frac{1}{2}-\frac{2}{9}=\frac{5}{18}
$$

$$
\begin{aligned}
& H(T x, T y)^{\frac{1}{\sqrt{H(T x, T y)}}}[d(x, y)+\min \{d(y, T x), d(x, T y)\}]^{-\frac{1}{\sqrt{d(x, y)+\min \{d(y, T x), d(x, T y)\}}}} \\
= & \left(\frac{1}{4}\right)^{2}\left(\frac{1}{2}-\frac{2}{n^{2}}\right)^{-\frac{1}{\sqrt{\frac{1}{2}-\frac{2}{n^{2}}}}} \\
\leq & \frac{1}{16}\left(\frac{18}{5}\right)^{\sqrt{\frac{18}{5}}} \\
< & \frac{1}{16}\left(\frac{18}{5}\right)^{2} \\
= & \frac{81}{100} .
\end{aligned}
$$

This shows that all conditions of Theorem 3 are satisfied and so $T \in \mathcal{M}_{W P}(X)$.

Acknowledgement 1. The authors would like to thank the referees for their helpful advice which led them to present this paper. 


\section{References}

[1] Ö. Acar, G. Durmaz and G. Minak, Generalized multivalued F-contractions on complete metric spaces, Bulletin of the Iranian Mathematical Society, 40 (6) (2014), 1469-1478.

[2] R. P. Agarwal, D. O'Regan and D. R. Sahu, Fixed Point Theory for Lipschitzian-type Mappings with Applications, Springer, New York, 2009.

[3] I. Altun, G. Durmaz, G. Minak and S. Romaguera, Multivalued almost F-contractions on complete metric spaces, Filomat, In press.

[4] I. Altun, G. Minak and H. Dağ, Multivalued F-Contractions On Complete Metric Space, Journal of Nonlinear and Convex Analysis, 16 (4) (2015), 659-666.

[5] V. Berinde, On the approximation of fixed points of weak contractive mappings. Carpathian J. Math. 19 (1) (2003), 7-22.

[6] V. Berinde, Iterative Approximation of Fixed Points, Springer-Verlag, Berlin Heidelberg, 2007.

[7] V. Berinde, Approximating fixed points of weak $\varphi$-contractions using the picard iteration, Fixed Point Theory, (2003), 131-147.

[8] V. Berinde, General constructive fixed point theorems for Ćirić-type almost contractions in metric spaces, Carpathian Journal of Mathematics, 24 (2) (2008), 10-19.

[9] M. Berinde and V. Berinde, On a general class of multivalued weakly Picard mappings, Journal of Mathematical Analysis and Applications, 326 (2007), 772-782.

[10] V. Berinde and M. Păcurar, The role of the Pompeiu-Hausdorff metric in fixed point theory, Creat. Math. Inform., 22 (2) (2013), 35-42.

[11] W.-S. Du, On coincidence point and fixed point theorems for nonlinear multivalued maps, Topology Appl., 159 (1) (2012), 49-56.

[12] W.-S. Du, Some new results and generalizations in metric fixed point theory, Nonlinear Anal., 73 (5) (2010), 1439-1446.

[13] V. I. Istrăţescu, Fixed Point Theory an Introduction, Dordrecht D. Reidel Publishing Company 1981.

[14] N. Mizoguchi and W. Takahashi, Fixed point theorems for multivalued mappings on complete metric spaces, J. Math. Anal. Appl.,141 (1989), $177-188$.

[15] G. Minak, M. Olgun and I. Altun, A new approach to fixed point theorems for multivalued contractive maps, Carpathian J. Math., 31 (2) (2015), 241-248. 
[16] S. B. Nadler, Multi-valued contraction mappings, Pacific J. Math., 30 (1969), 475-488.

[17] M. Olgun, G. Minak and I. Altun, A new approach to Mizoguchi-Takahashi type fixed point theorem, Journal of Nonlinear and Convex Analysis, In press.

[18] M. Pacurar, Iterative Methods for Fixed Point Approximation, Editura Risoprint, Cluj-Napoca, 2009.

[19] I. A. Rus, Basic problems of the metric fixed point theory revisited (II), Studia Universitatis Babeş-Bolyai Mathematica, 36 (1991), 81-99.

[20] I. A. Rus, A. Petrusel, A. Sintamarian, Data dependence of the fixed point set of some multi-valued weakly Picard operators, Nonlinear Anal. 52 (2003) 1947-1959.

[21] I. A. Rus, A. Petrusel, A. Sintamarian, Data dependence of the fixed points set of multivalued weakly Picard operators, Stud. Univ. Babeş-Bolyai, Math. 46 (2) (2001) 111-121.

[22] M. Sgroi and C. Vetro, Multi-valued F-contractions and the solution of certain functional and integral equations, Filomat, 27 (7) (2013), 1259-1268.

[23] X. Udo-Utun, On inclusion of $F$-contractions in $(\delta, k)$-weak contractions, Fixed Point Theory Appl. 2014, 2014:65, 6 pp.

[24] D. Wardowski, Fixed points of a new type of contractive mappings in complete metric spaces, Fixed Point Theory Appl. 2012, 2012:94, 6 pp.

Department of Mathematics, Faculty of Science and Arts, Kirikkale University, 71450 Yahsihan, Kirikkale, Turkey emails:g.minak.28@gmail.com, ishakaltun@yahoo.com

Instituto Universitario de Matemática Pura y Aplicada, Universidad Politécnica de Valencia, 46071 Valencia, Spain email:sromague@mat.upv.es 